\title{
A Relationship between Dispersion Measure and Redshift Derived in Terms of New Tired Light
}

\author{
Lyndon Ashmore \\ Independent Researcher, Dubai, UAE \\ Email: Lyndonashmore@outlook.com
}

How to cite this paper: Ashmore, L. (2016) A Relationship between Dispersion Measure and Redshift Derived in Terms of New Tired Light. Journal of High Energy Physics, Gravitation and Cosmology, 2, 512-530. http://dx.doi.org/10.4236/jhepgc.2016.24045

Received: June 17, 2016

Accepted: August 22, 2016

Published: August 25, 2016

Copyright (๑) 2016 by author and Scientific Research Publishing Inc. This work is licensed under the Creative Commons Attribution International License (CC BY 4.0).

http://creativecommons.org/licenses/by/4.0/

\section{(c) (i) Open Access}

\section{Abstract}

New data from FRB's have provided an exciting new window on the cosmos. For the first time we have both Dispersion Measure $(D M)$ from distant sources and their redshift. This gives us the opportunity to determine the average electron number density in intergalactic space and thus test New Tired Light predictions. Here, in an alternative cosmology, the universe is static and redshifts are produced by an interaction between photons and the electrons in the intergalactic medium. In a paper published in summer $2006 \mathrm{New}$ Tired Light (NTL) predicted an average electron number density of $n=0.5 \mathrm{~m}^{-3}$. In 2016 a paper was published reporting that for the first time the $D M$ of a FRB and the redshift of the host galaxy had been found. Using standard physics this confirmed the electron number density as $n=0.5 \mathrm{~m}^{-3}$. The prediction NTL made ten years earlier was proved to be correct. Using this measured electron number density enabled a definitive value of the Hubble constant to be made by New Tired Light and the value is $63 \mathrm{~km} / \mathrm{s}$ per Mpc which compares well with currently accepted values. Importantly, since in NTL the redshift and dispersion are both due to the electrons in IG space, a relationship between $D M$ s and redshift can be predicted. NTL predicts that $D M$ and $L N(1+\mathrm{z})$ will be directly proportional and related by the formula $D M=m_{e} c / 2 h r_{e}\left(3.086 \times 10^{22}\right)$ where $m_{e}, r_{e}$ are the rest mass and classical radius of the electron, $c$ is the speed of light in a vacuum and $h$ is the plank constant. The numerical term is to change units from $\mathrm{pccm}^{-3}$ to $\mathrm{m}^{-2}$. This reduces to $D M=2380 L N(1+z)$. Using data from five FRB's this is tested and a linear relation is seen of the form $D M=1830 L N(1+z)$. The gradient of the plot from the observed data is within $23 \%$ of that predicted by NTL. Recently the Tolman Surface Brightness test has been applied to the HUDF and the results support a static universe whilst the possibility of two differing types of SN Ia whose distribution changes with distances means that tired light models can no longer be ruled out. Using SDF we know the distance to the Atlia galaxy cluster as $1.26 \times 10^{24} \mathrm{~m}$. With the average electron number density of $n=0.5 \mathrm{~m}^{-3}$ found from the Dispersion Measures of the FRB's, from first principles, New Tired Light gives a calculated predicted 
redshift of 0.0086 . This compares well with the value found spectroscopically of 0.0087 -a difference of approximately $1 \%$. It is shown that if the energy transferred to a recoiling electron when a UV photon of wavelength $\lambda=5 \times 10^{-8} \mathrm{~m}$ interacts with it is emitted as a secondary photon that photon will have a wavelength of $2.2 \mathrm{~mm}-$ the wavelength at which the $\mathrm{CMB}$ curve peaks.

\section{Keywords}

Redshift, Tired Light, Hubble Constant, Expanding Universe, CMB, Supernovae Time Dilation

\section{Introduction}

The New Tired Light Theory (NTL) was first published in a peer reviewed journal in the summer of 2006 [1]. Here a prediction was made that in order for the NTL to give the correct value for the Hubble constant, the average electron number density, $n_{e}$ in intergalactic space would be $n_{e}=0.5 \mathrm{~m}^{-3}$. Until very recently it has been impossible to test this prediction precisely. However a paper published by Keane et al. [2] in February 2016 regarding FRB 150418 reported that "the Distance Measure of a FRB and host galaxy redshift have both been measured for the first time." The following data are given: redshift of host galaxy, $z=0.492 \pm 0.008$; Dispersion Measure, $D M=776.2(5) \mathrm{cm}^{-3}$; comoving distance $=1.88 \mathrm{Gpc}$; luminosity distance $=2.81 \mathrm{Gpc}$. It can be shown [3] that these data can be replicated using a cosmology calculator [4] and from it the average electron density along the line of sight can be determined. With input values of $H_{0}=69.6 \pm 1$ $\mathrm{km} / \mathrm{s}$ per $M p c$, Omega $\mathrm{M}=0.286$, Omegavac $=0.714$ and the redshift $z=0.492 \pm 0.008$. From this the light travel time is: $5.035 \pm 0.09 \mathrm{Gyr}$, or a distance of $1.543 \pm 0.03 \mathrm{Gyr}$.

Since the Distance Measure relationship is given by:

$$
D M=n_{e} d
$$

this leads to an average electron number density, $n_{e}$ of 0.5 electrons per cubic metre.

In NTL, the Hubble [1] constant is given by:

$$
H=2 n_{e} h r_{e} / m_{e} .
$$

Substituting values for $n_{e}, h, r_{e}$ and $m_{e}$ gives a value of $H=2.05 \times 10^{-18} \mathrm{~s}^{-1}$ which, in cosmological units is $63 \mathrm{~km} / \mathrm{s}$ per $M p c$ a difference of less than $10 \%$-thereby confirming the prediction made by NTL ten years earlier.

\section{Predicting a Relationship between $z$ and $D M$ by NTL}

The dispersion of radio waves is known to be produced by the interaction of the photons of the radiation with the electrons in space. In New Tired Light (NTL) it is proposed that the redshifting of light from distant galaxies is also produced by the interaction of photons of light with the same electrons in intergalactic space. Since it is the same electrons responsible for both effects one would expect a relationship between the 
Dispersion measure, $D M$ and the redshift, $z$.

The Dispersion Measure is the "integrated column density of free electrons between an observer and a source," and is given by the formula:

$$
D M=n_{e} d
$$

where $n_{e}$ is the average electron number density in $\mathrm{cm}^{-3}$ along line of sight and $\mathrm{d}$ is the distance from the source to observer in parsec.

In NTL, the redshift distance formula is given by (Appendix A; Equation (48)):

$$
z=\exp (H d / c-1)
$$

where $H$ is the Hubble constant, $c$ the speed of light in a vacuum, $d$ is the distance in metre and $z$ the redshift as before. Rearranging this equation to make $d$ the subject gives:

$$
d=\{c L N(1+z)\} / H .
$$

Equating the two equations gives:

$$
\left(3.086 \times 10^{22}\right)\left(D M / n_{e}\right)=\{c L N(1+z)\} / H .
$$

The term $3.086 \times 10^{22}$ being there to ensure both distances are in metre as $D M$ uses units of parsec per cubic centimetre and has to be converted.

In NTL the Hubble constant, $H$ is expressed in terms of a number of fundamental constants and the electron number density ([1], Appendix A; Equation (47)).

$$
H=2 n_{e} h r_{e} / m_{e}
$$

where $h$ is the plank constant and $r_{e}$ the classical electron radius.

Substituting this in the above gives:

$$
\left(3.086 \times 10^{22}\right)\left(D M / n_{e}\right)=\left\{m_{e} c L N(1+z)\right\} / 2 n_{e} h r_{e} .
$$

We see that the electron number density, $n_{e}$ cancels since for low electron densities any change in $n_{e}$ has the same effect on both the Distance Measure and redshift. This would not be true for large electron number densities since the electron will not recoil on absorption or re-emission and the redshift effect disappears but the dispersion effect continues. For dense plasma there will be dispersion but no redshift. Rearranging the equation for $D M$ gives:

$$
D M=\left\{\left(m_{e} c L N(1+z)\right) /\left[\left(3.086 \times 10^{22}\right)\left(2 h r_{e}\right)\right]\right\} .
$$

Inserting values for the constant terms i.e. $c=3 \times 10^{8} \mathrm{~m} / \mathrm{s}, m_{e}=9.1 \times 10^{-31} \mathrm{~kg}, h=$ $6.63 \times 10^{-34} \mathrm{~J} \cdot \mathrm{s}$ and $r_{e}=2.82 \times 10^{-15} \mathrm{~m}$ gives:

$$
D M=2380 L N(1+z) .
$$

We see that this is of the form $y=m x$ and so a graph of $D M$ against $L N(1+z)$ is a straight line passing through the origin with gradient $2380 \mathrm{~m}^{-2}$.

This gives us the opportunity to test the New Tired Light Theory. Will a graph of 
$D M$ versus $L N(1+z)$ be a straight line through the origin and importantly how close is the gradient to this combination of Universal constants as predicted by NTL?

Thornton in 2013 [5] reported both $D M$ and $z$ for four FRB's emitted from pulsars and Keane et al. [2] in 2016 reported one $D M$ and $z$ from a radio transient in a host galaxy. Figure 1 shows a plot of the data. Note the discrepancy between the two sets of observed data and that the Thornton data does not pass through the origin but has a significant intrinsic redshift of 0.078 when the $D M$ is zero.

It is proposed that since the Thornton data is for pulsars i.e. neutron stars, that there will be a significant gravitational redshift and that this is the cause of this intrinsic redshift. Pulsars can have gravitational redshifts of up to 0.6 [6] and so it is quite possible that this is the cause of the intrinsic redshifts in the pulsar data. In Figure 2 this intrinsic redshift has been removed and we immediately see a good correlation between the two sets of data. Additionally there is good agreement between the shape of the two graphs for the Thornton data and that predicted by NTL. The equation of the line in the gravitationally redshifted adjusted Thornton data is:

$$
D M=1830 L N(1+z)
$$

which compares well with the NTL prediction of:

$$
D M=2380 L N(1+z) .
$$

The percentage difference between the gradient for the observed data and that

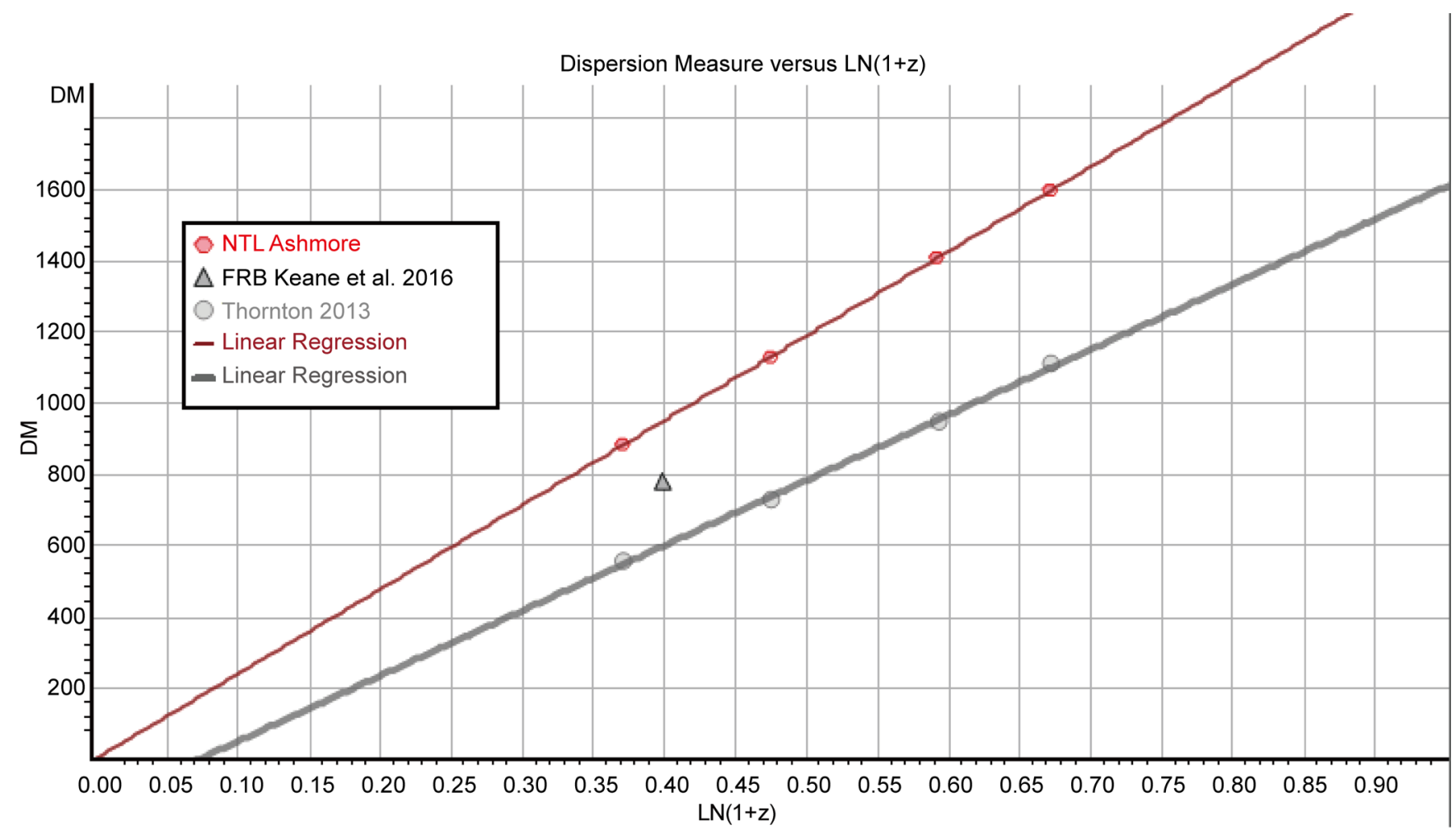

Figure 1. Dispersion measure versus $L N(1+z)$ for the Thornton pulsar data (grey), the Keane data point and the predicted relationship given by NTL (red). 


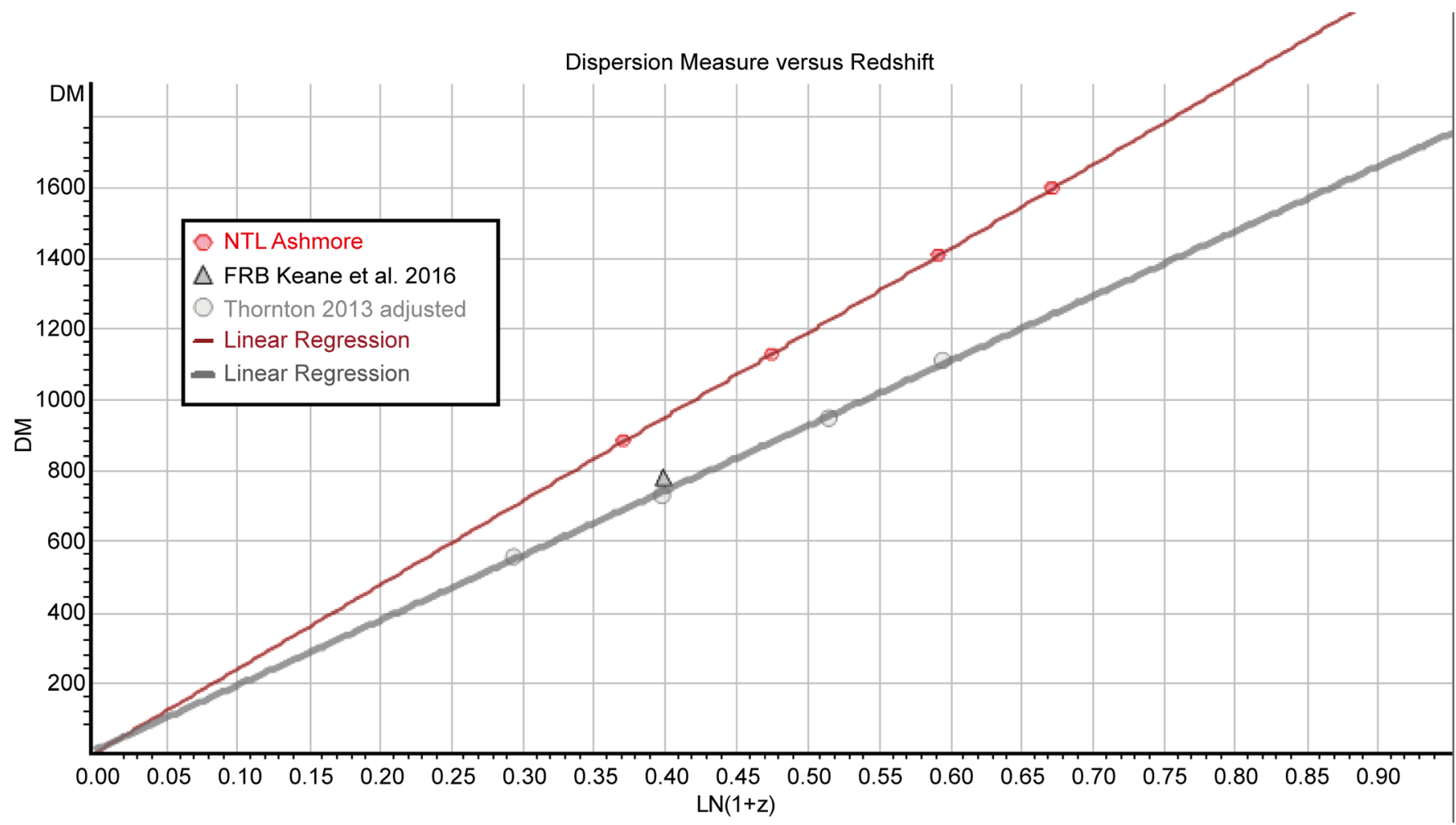

Figure 2. Dispersion measure versus $L N(1+z)$ with the Thornton pulsar data adjusted for a possible intrinsic gravitational redshift of 0.078. Note how the Thornton pulsar data now agrees with the Keane FRB data.

predicted by NTL is $23 \%$. If dispersion is an effect of a photon electron interaction whilst redshift, in mainstream cosmology, is an effect due to the stretching of space then there may well be some qualitative agreement between the two but here we have the same linear graph and the gradient of the observed DMs within $23 \%$ of that predicted by NTL. Remember, that this same gradient predicted by NTL is a combination of universal constants. Why is it that the constant of proportionality between two purportedly "unrelated properties" just happens to be a combination of the radius and mass of the electron, the speed of light and the plank constant? If true it is a very remarkable coincidence indeed.

\section{Tests to Distinguish between Static and Expanding Models of the Universe}

The Big Bang Theory is not the only theory of the Universe. There are many others as well as New Tired Light. For instance the cosmological data can be, in principle, explained through extended theories of gravity, as it is stressed, for example, in [7]. Several tests have been put forward to discriminate between static and expanding cosmologies.

\subsection{Tolman Surface Brightness Test}

The surface brightness of distant galaxies should fall off with increased redshift in a 
predicted way: that is brightness is proportional to $(1+z)^{-n}$. Importantly, the predicted relationship is different for the two models. In a static universe " $n$ " has the value of 1 whilst in an expanding universe " $n$ " has the value of 4 [8]. A test performed in 2001 [9] found the value of $n$ to be $2.59 \pm 0.17$ in the "R band" and $3.37 \pm 0.13$ in the "I band" and claimed the results ruled out a static universe but not an expanding one. One problem with the Tolman test is that it does not compare like with like and does not take into account any evolution of galaxies. Whilst the brightness of both galaxies is compared here on Earth at the same time the light from the more distant galaxy set off long before that from the nearer one i.e. it could be that the more distant galaxy was younger than the nearer one when the light set off and the results are due to evolution of the galaxies concerned rather than the cosmological model. That said, the results are not spectacularly convincing at first sight with 2.59 in the " $\mathrm{R}$ band" which a cynic may say is more like "splitting the difference" between the two models rather than distinguishing between them. To resolve the issue Lerner et al. [10] looked at the UV surface brightness of galaxies from the local Universe to $z \sim 5$ using the HUDF and GALEX datasets. They concluded "that available observations of galactic $S B$ are consistent with a static Euclidean model of the Universe."

\subsection{Supernovae Light Curves}

Another test of the differing cosmologies are supernovae Ia light curves [11]. These are graphs of intensity versus time and are reported to increase in width as the redshift increases. That is, the further away the supernova event, the broader the light curve becomes. In an expanding Universe this is explained as relativistic time dilation and is said to support the idea of an expanding universe. However, it must be remembered that what is measured is that "the further away the supernovae, the longer it takes to reach maximum brightness and then fade." This could be caused by a Malmquist bias [12] as the width of the Supernovae light curves do increase as the brightness increases [13]. As we look further and further into the distance we need to find brighter and brighter supernova events as the dimmer ones will no longer be able to be seen. Since the more distant supernova observed will be brighter they will have a broader light curve. Hence the observed result.

If indeed relativistic effects were responsible for the curve broadening then one would expect to see broadening of quasar light curves and one doesn't. Various groups have looked at quasar light curves from quasars at high and low redshift and can find no evidence of any broadening [14]-[17]. These results thus support a static Universe and rule out expansion.

How is it that supernovae light curves are said to display time dilation but quasar light curves do not-and over the same range of redshifts? One possible answer is dispersion. Different frequencies of light travel at different speeds in the intergalactic medium. A supernova is a short sharp pulse of light and, just as a multi-colour digital pulse broadens as it travels down a fibre optic, the supernova pulse will broaden as it travels through space. The further they travel the broader they become. The quasar 
light curves are not pulses but continuous and so we would not see any effects due to dispersion. Until the two light curve effects of supernovae and quasars are explained the time dilation explanation should be treated with caution. This is particularly so in respect of the newly discovered evidence that not all supernovae events are identical. It was thought that a supernovae Ia explosion was caused by accretion from a nearby companion star and thus identical but a recent paper [18] discounts this explanation and suggests they are caused by the merger of binary white stars or other compact stars. This shows that our understanding of SN Ia's is not complete. It must be said that it does appear that whilst they don't all start off the same way they still appear to decay in the same way.

One way to test the supernovae data would be to look at the number density of supernovae over a range of redshifts. In an expanding universe as we look back in time the universe was smaller then and so the supernovae events, on average, should be closer together in redshift as the redshift increases. In a static universe the supernova should, on average, be evenly distributed in redshift. It appears that this has not been done before so let us look at the data. The "Union 2.1" SNIa Compilation Magnitude vs. Redshift Table at the supernova cosmology project [19] gives a compilation of data from several datasets and includes $833 \mathrm{SNe}$ drawn from 19 datasets. Of these $580 \mathrm{SNe}$ Ia pass usability tests. The 580 SN Ia were placed in bins of redshift size 0.05 and a SN Ia number density versus redshift derived (Figure 3 ).

Whilst this is just a simple test, the high number of SN Ia's between $z=0-0.05$ compared to the much lower number distribution at a higher redshift is striking. Note also that for redshifts above 0.05 the distribution is fairly flat and consistent with a

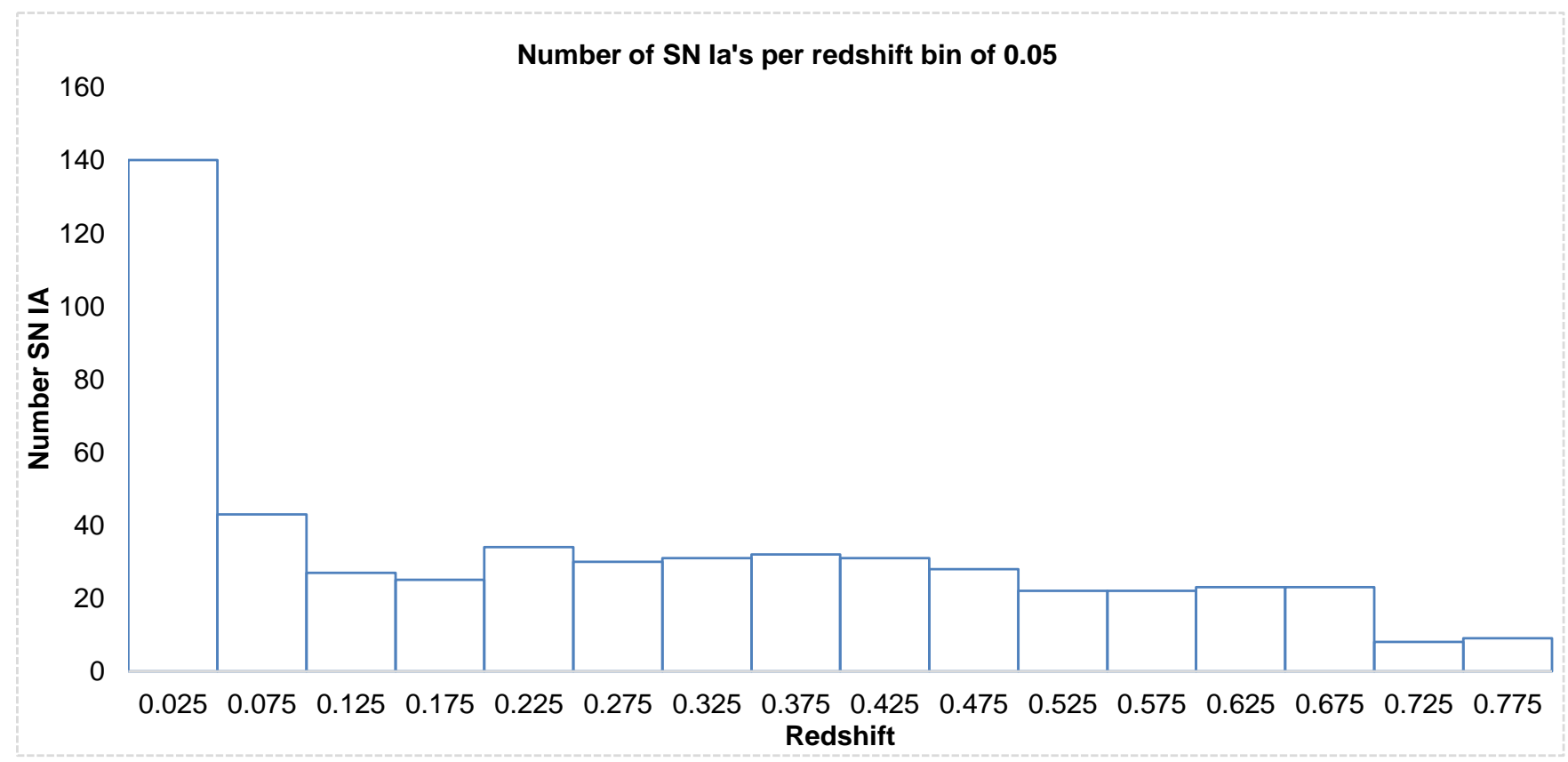

Figure 3. Number-density evolution of the Supernovae Ia from the "Union2.1" SNIa Compilation Magnitude vs. Redshift Table at the supernova cosmology project. 
static universe. Could it be that there are two types of SN Ia's-with one set not as bright as the second type and so only "local" ones are seen of the first type? If so, this would have an impact on the broadening of light curves since the local SN Ia's are used to set the templates on the basis that they are said to have negligible broadening. These are then used on the more distant ones to measure how stretched they are. In 2015 [20] it was reported that there were two types of SN Ia which they labelled NUV-red and NUV-blue (NUV-Near Ultra Violet) and their luminosities vary in the UV section of the spectrum indicating that they may be caused by different events. Importantly, in the nearby Universe almost 70\% of SN Ia's are NUV-red and farther away the percentages fall such that only $10 \%$ of SN Ia's are now NUV-red. This would imply that a light curve template found from an average of nearby SN Ia's would not be valid when applied to the more distant ones.

\section{Predicting Redshift from First Principles}

It is often said that "the proof of the pudding is in the eating," so let us calculate the redshift of the Antlia Cluster (or Abell S0636) from First principles.

The galaxy cluster Antlia is chosen for no other reason than its' distance and redshift are known and measured using different, separate techniques and it is close enough for us to consider the collision cross-section constant throughout its journey. The distances to far off galaxies are often taken from their redshift as they are too far away for standard candles to be used and this would then not be a fair test of NTL. Additionally, the galaxy must be far enough away for it to be in the "Hubble flow" where any gravitational effects are insignificant when compared to the cosmological redshift. The distance, $d$ to Antlia is known from Surface Brightness Fluctuations (SBF) as 132.7 ly [21] or $1.26 \times$ $10^{24} \mathrm{~m}$. The SBF method of determining distances to galaxies uses the idea that the surface brightness of nearby but unresolved star clusters or galaxies appear "bumpy", whilst those of distant ones appear "smooth." By looking at the Poisson fluctuations in the number of unresolved stars within a single CCD pixel one can quantify the effect and determine the distance [22].

To predict the redshift we will consider a photon of light of wavelength $\lambda=5 \times 10^{-7}$ $\mathrm{m}$ travelling to the Earth.

The collision cross-section, $\sigma$ is (Appendix A, Equation (35)):

$$
\sigma=2 r_{e} \lambda=2.82 \times 10^{-21} \mathrm{~m}^{2} .
$$

The mean free path, $l$ is:

$$
l=\left(2 n_{e} r_{e} \lambda\right)^{-1}=7.10 \times 10^{20} \mathrm{~m} .
$$

In light-year, the mean free path is approximately 75,000 ly or to put it another way, our photon of light only interacts with an electron in space, on average, every 75,000 years on its journey.

Using the value of $n_{e}=0.5 \mathrm{~m}^{-3}$ as derived earlier from Dispersion Measure techniques.

The number of interactions, $N$ suffered by the photon on its journey is the total dis- 
tance divided by the mean free path. Or:

$$
N=d / l=1774 \text {. }
$$

That is, photons of this wavelength will be, on average, absorbed and re-emitted 1774 times on their journey through space.

In NTL, on each interaction the wavelength is increased by $\delta \lambda=h / m_{e} c=2.42 \times 10^{-12}$ $\mathrm{m}$ (Appendix A, Equation (34)) and so the total increase in wavelength, $\Delta \lambda$ is:

$$
\Delta \lambda=N \times 2.42 \times 10^{-12}=4.29 \times 10^{-9} \mathrm{~m} .
$$

The redshift, $z$ is:

$$
Z=\Delta \lambda / \lambda=\left(4.29 \times 10^{-9}\right) /\left(5 \times 10^{-7}\right)=0.0086
$$

The spectroscopically measured average redshift of the cluster is 0.0087 [23] a difference between the predicted value by NTL and the observed value of just over $1 \%$.

In NTL the Hubble relationship becomes "photons of light from a galaxy twice as far away, travel twice as far through the Intergalactic medium, interact with twice as many electrons experience twice the increase in wavelength and thus undergo twice the redshift."

Note that in this interaction, the recoil takes place along the direction of the line of sight and so there will be no "blurring" of the image as in the Compton Effect.

The energy transferred to the recoiling electron is emitted as a secondary photon which forms the $C M B$. Two secondary photons are emitted, one on absorption and one on re-emission so let us calculate the wavelength of these photons and compare it to the $C M B$ spectrum:

Momentum of incoming photon is $h / \lambda$ where $h$ and $\lambda$ are the planck constant and the wavelength of the incoming photon respectively. By conservation of momentum:

$$
h / \lambda=m_{e} v
$$

where $m_{e}$ is the electron rest mass and $v$ is the recoil velocity giving $v=1460 \mathrm{~ms}^{-1}$.

The Kinetic energy of the recoiling electron is:

$$
E_{k}=m_{e} v^{2} / 2=9.66 \times 10^{-25} \mathrm{~J} .
$$

This energy is radiated as a secondary photon of frequency $f$ given by:

$$
E=h f_{C M B} \text { or } f_{C M B}=9.66 \times 10^{-25} / h=1.46 \times 10^{9} \mathrm{~Hz} .
$$

The wavelength $\lambda_{C M B}$ is:

$$
\lambda_{C M B}=c / f_{C M B}=21 \mathrm{~cm} .
$$

We see that the wavelength of the secondary radiation is in the microwave region. However a little longer than the peak of the $C M B$ spectrum implying that the majority of the photons of the $C M B$ are produced by UV.

In any case, we have only shown the NTL theory for one wavelength in the visible region of the e-m spectrum. Redshift, $z$ is a constant throughout the spectrum so let us repeat the exercise for a UV photon of wavelength, $\lambda=5 \times 10^{-8} \mathrm{~m}$.

The collision cross-section, $\sigma$ is (Appendix A, Equation (35)): 


$$
\sigma=2 r_{e} \lambda=2.82 \times 10^{-22} \mathrm{~m}^{2}
$$

The mean free path, $l$ is:

$$
l=\left(2 n_{e} r_{e} \lambda\right)^{-1}=7.10 \times 10^{21} \mathrm{~m}
$$

In light-year, the mean free path is approximately 750,000ly or to put it another way, our UV photon only interacts with an electron in space, on average, every three quarters of a million year on its journey.

Using the value of $n_{e}=0.5 \mathrm{~m}^{-3}$ again.

The number of interactions, $N$ suffered by the photon on its journey is the total distance divided by the mean free path. Or:

$$
N=d / l=177 \text {. }
$$

That is, photons of this wavelength (UV) will be, on average, absorbed and re-emitted 177 times on their journey through space. In NTL, on each interaction the wavelength is increased by $\delta \lambda=h / m_{e} c=2.42 \times 10^{-12} \mathrm{~m}$ (Appendix A, Equation (34)) and so the total increase in wavelength, $\Delta \lambda$ is:

$$
\Delta \lambda=N \times 2.42 \times 10^{-12}=4.28 \times 10^{-10} \mathrm{~m} .
$$

The redshift, $z$ is:

$$
Z=\Delta \lambda / \lambda=\left(4.29 \times 10^{-10}\right) /\left(5 \times 10^{-8}\right)=0.0086
$$

The same redshift as before showing that in NTL redshift, $z$ is independent of the wavelength as required from observation. The reason for this is that in NTL, a photon with twice the wavelength, $\lambda$ has twice the collision cross-section, undergoes twice the number of interactions with the electrons in IGM when travelling the same distance, experiences twice the increase in wavelength, $\Delta \lambda$ such that the redshift $z=\Delta \lambda / \lambda$ remains constant.

Now let us look at the secondary radiation given off by this UV photon when it interacts with the electrons in the IGM. By conservation of momentum,

$$
h / \lambda=m_{e} v
$$

where $m_{e}$ is the electron rest mass and $v$ is the recoil velocity giving $v=14,548 \mathrm{~ms}^{-1}$.

The Kinetic energy of the recoiling electron is:

$$
E_{k}=m_{e} v^{2} / 2=9.64 \times 10^{-23} \mathrm{~J} .
$$

This energy is radiated as a secondary photon of frequency $f$ given by:

$$
E=h f_{C M B} \text { or } f_{C M B}=9.66 \times 10^{-25} / h=1.45 \times 10^{11} \mathrm{~Hz} .
$$

The wavelength $\lambda_{C M B}$ is:

$$
\lambda_{C M B}=c / f_{C M B}=2.1 \mathrm{~mm}
$$

Not only is this in the microwave region but it is the peak wavelength of the $C M B$ [24] [25]. We see that the bulk of the $C M B$ is formed by secondary radiation given off by photons in the UV as they are redshifted by NTL. This also explains why there are just so many $C M B$ photons in the universe. If our photon of wavelength $\lambda=5 \times 10^{-8} \mathrm{~m}$ 
were to undergo a redshift of 0.1 then:

$$
\begin{aligned}
& z=\Delta \lambda / \lambda \\
& 0.1=\Delta \lambda / 5 \times 10^{-8} \\
& \Delta \lambda=5 \times 10^{-9} \mathrm{~m}
\end{aligned}
$$

On each interaction the shift in wavelength is $\delta \lambda=h / m_{e} c=2.42 \times 10^{-12} \mathrm{~m}$ and in undergoing a total redshift of 0.1 it would interact with an electron $5 \times 10^{-9} / 2.42 \times 10^{-12}=$ 2066 times producing two $C M B$ photons each time thus giving us a total of $4132 C M B$ photons from a single UV photon-and this for a redshift of just 0.1 .

\section{Discussion}

We are told that a true test of a theory is that it should predict something that is later proved to be correct. With the Big Bang theory and the Steady State theory, the Big Bang theory predicted the $C M B$ but the Steady State theory did not. The discovery of the $C M B$ is considered to be support of the Big Bang because it predicted this radiation and it was later found. So what of New Tired Light? It predicted in a peer reviewed journal that the average electron number density in intergalactic space would be 0.5 $\mathrm{m}^{-3}$. Ten years on with the Keane et al.'s paper we can measure this using standard, mainstream physics and we find the value is $0.5 \mathrm{~m}^{-3}$ exactly as predicted by NTL.

With this measured value of the average electron number density in intergalactic space of $0.5 \mathrm{~m}^{-3}$, NTL predicts a Hubble constant of $63 \mathrm{~km} / \mathrm{s}$ per Mpc. The error between this value and the oft quoted value of $H_{0}=69.6 \pm 1 \mathrm{~km} / \mathrm{s}$ per Mpc is less than $10 \%$. However some reputable scientists and places of reference give the Hubble constant as $64 \mathrm{~km} / \mathrm{s}$ per Mpc [26] an error between the NTL prediction and measurement of less than 2\%. Can the Big bang theory do this? That is to predict a value for the Hubble constant, use accepted physics principles and actual measured data to predict a value for the Hubble constant that is within $2 \%$ of the measured value-and do this ten years in advance of all the data being available?

An exciting new area of cosmology is FRB's. Due to dispersion effects in the radio pulses from these short bursts we are not only able to find the average electron number density along the line of sight but we can explore the relationship between Dispersion Measure $(D M)$ and redshift. It is now a possibility to use $D M \mathrm{~s}$ as a distance measure in cosmology. In the Big Bang theory there is a qualitative relationship between the twoas redshift increases so too will the $D M$. However, since $D M$ s are due to average electron number density and redshift is due to a stretching of space one would not expect a simple mathematical relationship between the two. As the Universe expands the number density should reduce as the radio photons travel towards us. In mainstream cosmology the universe is accelerating so one would not expect a simple linear relationship to occur. NTL not only predicts a linear proportional relationship between $D M$ and $L N(1+z)$ but also tells us that a graph of $D M$ versus $L N(1+z)$ will be a straight line through the origin and tells us that the gradient will be a combination of universal constants. Using data from a set of four FRB's we are able to construct a graph of $D M$ ver- 
sus redshift. NTL predicted a gradient of $2380 \mathrm{~m}^{-2}$ whilst the observations give a value of $1830 \mathrm{~m}^{-2}$. This is a difference of $23 \%$ between the observed value and that predicted by NTL-and remember this is not any number but a combination of several universal constants. What is the probability of this happening by chance as the Big Bang theory would have us believe?

Tests have been developed to discriminate between models of the Universe that are expanding and those that have a static universe. One of the first is the Tolman Surface Brightness test which had at first favoured an expanding Universe. However, when applied recently to the HUDF for redshifts over a wide range from local to $z \sim 5$ we see that it supports a static universe. Curve broadening of SN Ia's are said to be caused by relativistic effects due to expansion. If so why do Quasar light curves not show any such effects? If curve broadening is due to the intervening space between source and observer stretching then the source should not matter. Either the light curves from SN Ia's and quasars should both be stretched or neither. These two conflicting results must be reconciled before light curve broadening can be considered as evidence of expansion. Unless curve broadening is caused by something else such as dispersion of the multicolour pulses from SN Ia whilst the continuous curves from quasars would not show it. Or could it be a Malmquist bias? In the study of SN Ia light curves local ones are used to find a mean light curve and then this is used on the more distant ones which are then said to be broadened. Recent observations show that there are two types of SN Ia which have differing intensities. Importantly, in the near region where SN Ia's are used to find the mean light curve template $70 \%$ of the sample are NUV-red. When this template is applied to distant ones the sample contains only $10 \%$ of NUV-red. Could it be that the curve broadening is just a gradual change in the distribution of NUV-red to NUV-blue as the distance increases?

Then there is the problem of the $C M B$ [1]. NTL states that energy is lost to the recoiling electron and this is then re-emitted as a secondary photon which forms the $C M B$. These photons are in the microwave region. How is it that a photon of UV when redshifted produces a secondary radiation with photons at the peak of the $C M B$ curve? Plasma is known to emit black body radiation.

There still remains the problem of average Hydrogen cloud separation [27]. How is it that on average Hydrogen clouds manage to be evenly spaced for more than the last billion years and yet, allegedly, the space in which they exist is expanding carrying them apart?

As we look back in time they should be getting closer and closer as we rewind the expansion. In an expanding universe the clouds should be getting further and further apart as the universe ages. That is the number of Hydrogen clouds per unit redshift should decrease as the redshift $z$ decreases. But they don't. We see that the hydrogen clouds are evenly spaced up to a redshift of approximately 0.35 (see Figure 4) [28] implying a static universe for the last billion year or so. This is in agreement with the mean separation of SN Ia's at least up to a redshift of approximately 0.5 .

Using NTL from first principles to calculate the redshift of a galaxy at a known 


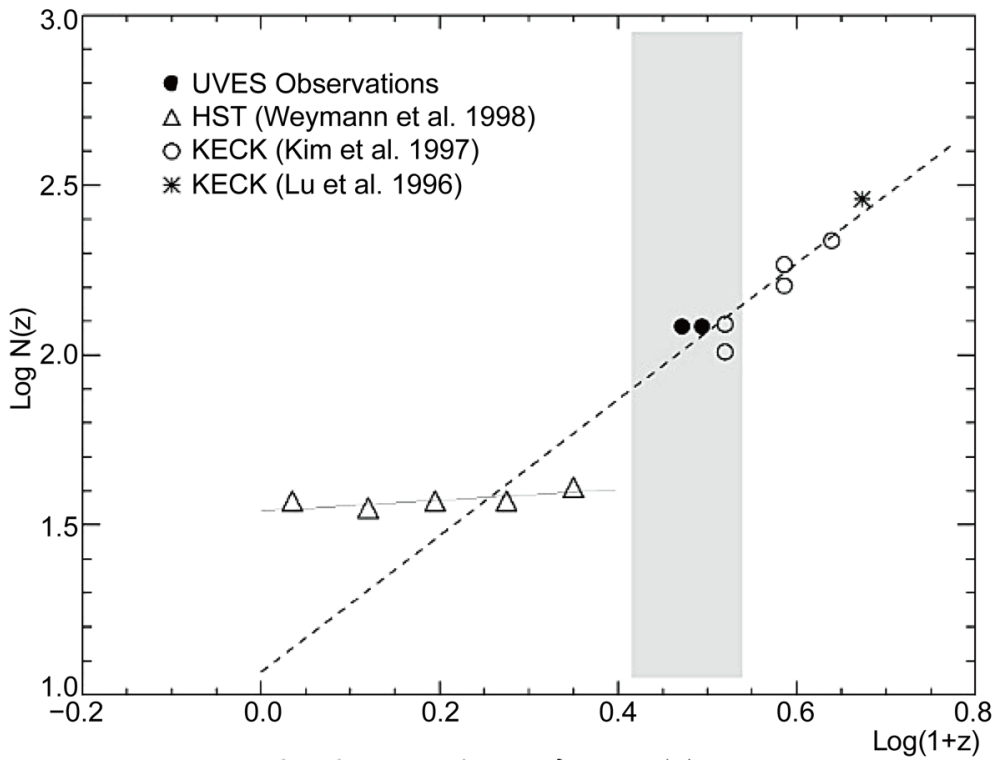

Figure 4. This image shows the number-density evolution of the Lyman alpha lines, observed with UVES in the quasars HE22-28 and QSO J2233-606. Credit: ESO

https://www.eso.org/public/images/eso0013g/.

distance determined from its' SBF using NTL ideas gives an answer differing by only $1 \%$ from the observed value gives support to New Tired Light. Here we have four completely different areas of Physics and yet they agree totally. SBF is used to determine the distance to a galaxy cluster, the Dispersion Measure is used to determine the mean electron number density, NTL uses both of these results to predict the redshift of the cluster. The redshift is measured spectroscopically and gives an answer differing by just $1 \%$. What is the probability of that happening by mere chance? It gives great support in favour of New Tired Light.

\section{References}

[1] Ashmore, L.E. (2006) Recoil between Photons and Electrons Leading to the Hubble Constant and CMB. Galilean Electrodynamics, 17, 53.

[2] Keane, E.F., Johnston, S., Bhandari, S., Barr, E., Bhat, N.D.R., Burgay, M., et al. (2016) The Host Galaxy of a Fast Radio Burst. Nature, 530, 453-456. http://dx.doi.org/10.1038/nature17140

[3] Kiernein, J and Marmet, L. (2016) Private Emails.

[4] http://www.astro.ucla.edu/ wright/CosmoCalc.html

[5] Thornton, D., Thornton, D., Stappers, B., Bailes, M., Barsdell, B., Bates, S., Bhat, N.D.R., et al. (2013) A Population of Fast Radio Bursts at Cosmological Distances. Science, 341, 53-56. http://dx.doi.org/10.1126/science.1236789

[6] Aspel, D. (2001) Pulsars and Redshifts. http://arxiv.org/abs/gr-qc/0104025

[7] Corda, C. (2009) Interferometric Detection of Gravitational Waves: The Definitive Test for General Relativity. International Journal of Modern Physics D, 18, 2275. http://dx.doi.org/10.1142/S0218271809015904

[8] Geller, M.J. and Peebles, J.E. (1972) Test of the Expanding Universe Postulate. Astrophysical 
Journal, 174, 1-5. http://dx.doi.org/10.1086/151462

[9] Lubin, L.M. and Sandage, A. (2001) The Tolman Surface Brightness Test for the Reality of the Expansion. IV. A Measurement of the Tolman Signal and the Luminosity Evolution of Early-Type Galaxies. Astronomical Journal, 122, 1084-1103.

http://dx.doi.org/10.1086/322134

[10] Lerner, E.J., Falomo, R. and Scarpa, R. (2014) UV Surface Brightness of Galaxies from the Local Universe to $z \sim 5$. International Journal of Modern Physics D, 23, Article ID: 1450058. http://dx.doi.org/10.1142/s0218271814500588

[11] Blondin, S., Davis, T.M., Krisciunas, K., Schmidt, B.P., Sollerman, J., Wood-Vasey, W.M. and 26 Co. Authors (2008) Time Dilation in Type Ia Supernova Spectra at High Redshift. The Astrophysical Journal, 682, 724-736. http://dx.doi.org/10.1086/589568

[12] Philips, M.M. (1993) The Absolute Magnitudes of Type Ia Supernovae. The Astrophysical Journal, 413, L105-L108. http://dx.doi.org/10.1086/186970

[13] Kasen, D. and Woosley, S.E. (2007) On the Origin of the Type Ia Supernova Width-Luminosity Relation. The Astrophysical Journal, 656, 661-665. http://dx.doi.org/10.1086/510375

[14] Hook, I.M., McMahon, R.G., Boyle, B.J. and Irwin, M.J. (1994) The Variability of Optically Selected Quasars. Monthly Notices of the Royal Astronomical Society, 268, 305-320. http://dx.doi.org/10.1093/mnras/268.2.305

[15] Cristiani, S., Trentini, S., La Franca, F., Aretxaga, I., Andreani, P., Vio, R., Gemmo, A., et al. (1996) The Optical Variability of QSOs. Astronomy and Astrophysics, 306, 395-407.

[16] Hawkins, M.M. (2001) Time Dilation and Quasar Variability. The Astrophysical Journal, 553, L97-L100. http://dx.doi.org/10.1086/320683

[17] Hawkins, M.M. https://arxiv.org/abs/1004.1824

[18] Milne, P.A., Brown, P.J. and Narayan, G. (2015) The Changing Fractions of Type Ia Supernova NUV-Optical Subclasses with Redshift. The Astrophysical Journal, 803, 20.

[19] http://supernova.lbl.gov/Union/figures/SCPUnion2.1_mu_vs_z.txt

[20] Milne, P.A., Foley, R.J., Brown, P.J. and Narayan, G. (2015) The Changing Fractions of Type Ia Supernova Nuv-Optical Subclasses with Redshift. The Astrophysical Journal, 803, 20. http://dx.doi.org/10.1088/0004-637X/803/1/20

[21] Tonry, J.L., et al. (2001) The SBF Survey of Galaxy Distances. IV. SBF Magnitudes, Colors, and Distances. The Astrophysical Journal, 546, 681-693. http://dx.doi.org/10.1086/318301

[22] Blakeslee, J.P., et al. (1999) Distances from Surface Brightness Fluctuations. ASSL, 237, 181B.

[23] Nakazawa, K., Makishima, K., Fukazawa, Y. and Tamura, T. (2000) ASCA Observations of a Near-By Cluster in Antlia. Publications of Astronomical Soc of Japan, 52, 623-630. http://dx.doi.org/10.1093/pasj/52.4.623

[24] Peebles, P.J.E., Schramm, D.N., Kron, R.G. and Yurner, E.L. (1991) The Case for the Relativistic Hot Big Bang Cosmology. Nature, 352, 769-776.

[25] Nagano, M. and Watson, A.A. (2000) Observations and Implications of the UltrahighEnergy Cosmic Rays. Reviews of Modern Physics, 72, 689-732. http://dx.doi.org/10.1103/RevModPhys.72.689

[26] http://www.astro.ucla.edu/ wright/cosmo_01.htm

[27] Ashmore, L.E. (2009) Hydrogen Cloud Separation as Direct Evidence of the Dynamics of the Universe. $A S P C, 413,3$.

[28] ESO. https://www.eso.org/public/images/eso0013g/ 
[29] Ashmore, L.E. (2016) An Explanation of Redshifts in a Static Universe. In: Amoroso, R.L., Kauffman, L.H. and Rowlands, P., Eds., Unified Field Mechanics, World Scientific Publishers, Singapore, 456-462.

[30] French, A.P. (1968) Special Relativity. Nelson, London, 128.

[31] Feynman, R. (1990) QED—The Strange Story of Light and Matter. Penguin, London, 76.

[32] Berestetskii, V.B., Lifshitz, E.M. and Pitaevskii, L.P. (1982) Quantum Electrodynamics. Volume 4, 2nd Edition, Butterworth Heinemann, Oxford, 161-221.

[33] Mitchner, M. and Kruger, C.H. (1973) Partially Ionized Gases. Wiley, Hoboken, 138.

[34] Kurth, W.S. http://www.physics.uiowa.edu/plasma-wave/tutorial/waves.html

[35] Cairns, R.A., Ersfeld, B., Johnson, D., McDonald, D.C. and Ruhl, H. (1998) Nonlinear Harmonic Response in Laser-Plasma Interactions. Physica Scripta, T75, 99-103. http://dx.doi.org/10.1238/Physica.Topical.075a00099

[36] Zombeck, M.V. (2010) Handbook of Space Astronomy and Astrophysics. Cambridge University Press, Cambridge, 286. http://ads.harvard.edu/books/hsaa/idx.html

[37] French, A.P. (1968) Special Relativity. Nelson, London, 176-182.

[38] Henke, B.L., Gullikson, E.M. and Davis, J.C. (1993) X-Ray Interactions: Photoabsorption, Scattering, Transmission, and Reflection at $E=50-30,000 \mathrm{eV}, \mathrm{Z}=1$ - 92. Atomic Data and Nuclear Data Tables, 54, 181-342. http://dx.doi.org/10.1006/adnd.1993.1013

[39] Henke, B.L., Gullikson, E.M. and Davis, J.C. (2001) X-Ray Data Booklet. Chapter 1, LBNL/PUB-490, Rev. 2, Lawrence Berkeley National Laboratory, University of California, Berkeley, 44-52. http://www-xro.lbl.gov/optical_constants/intro.html

[40] Hubbell, J.H., Veigele, W.J., Briggs, E.A., Brown, R.T., Cromer, D.T. and Howerton, R.J. (1975) Atomic Form Factors, Incoherent Scattering Functions, and Photon Cross Sections. Journal of Physical and Chemical Reference Data, 4, 471-538. http://dx.doi.org/10.1063/1.555523

[41] Zwicky, F. (1929) On the Red Shift of Spectral Lines through Interstellar Space. Proceedings of the National Academy of Sciences of the United States of America, 15, 773-785. http://dx.doi.org/10.1073/pnas.15.10.773

[42] Platonov, K.Y. and Fleishman, G.D. (2002) Transition Radiation in Media with Random Inhomogeneities. Physics-Uspekhi, 45, 235-291. http://dx.doi.org/10.1070/PU2002v045n03ABEH000952

[43] Berestetskii, V.B., Lifshitz, E.M. and Pitaevskii, L.P. (1982) Quantum Electrodynamics. Vol. 4, 2nd Edition, Butterworth Heinemann, Oxford, 389. 


\section{Appendix A. Review of New Tired Light}

What follows is a review of previously papers on New Tired Light [1] [29].

\section{A.1. The Hubble Constant}

When light travels through a transparent medium it does so by being constantly absorbed and re-emitted by the electrons in the atoms of that medium [30] [31]. In resonance absorption the energy of the photon is transferred to the oscillating system, there is a delay and then the energy is re-emitted as a new photon. Since electrons in the plasma of the Intergalactic medium (IGM) can perform SHM [32] [33] they too can absorb and re-emit photons of light. Since there is a delay between absorption and reemission the speed of light in a medium is less than that in a vacuum. In a transparent medium such as glass the electrons are "fixed" in the atoms which are in turn "fixed" in the block of glass and so any recoil of the electrons is negligible since it is the whole block of glass that recoils. There is no energy loss to the photon in the glass and hence no redshift. However, since the IGM is sparsely populated, the electrons in the plasma absorb the photon, the energy of the photon is transferred to the oscillating electron which also recoils. The energy transferred to the recoiling electron is "lost" to the electron and is emitted as a secondary photon (we shall see later that it is these secondary electrons that form the $C M B$ ). The energy stored in the oscillating electron is re-emitted as a "new" photon-but not all of it as the electron recoils again on re-emission. This recoil energy is also emitted as a secondary photon and since two photons are emitted in the process the photon is able to continue in a straight line. There will be no blurring of an image. Since in NTL, the recoil acts along the line of sight and not across it.

Provided the frequency of the photon is well away from the resonant frequency of the electron in the plasma (which is the same as the plasma frequency) [34] [35] then the photon will always be re-emitted and since the plasma frequency in IGM is less than $30 \mathrm{~Hz}$ [36] this is the case. Since the photon loses energy at each interaction with the electrons in the plasma the frequency of the photon is reduced and the wavelength increased. It has been redshifted.

The energy transferred to an electron by recoil is known [37] and $=Q^{2} / 2 m_{e} c^{2}$ where $Q$ is the photon energy $m_{e}$ the electron rest mass and $\mathrm{c}$ the speed of light. Since energy is lost on both absorption and re-emission we must apply this twice so total energy lost per interaction $=Q^{2} / 2 m_{e} c^{2}=h^{2} c^{2} / \lambda m_{e}^{2}$ or:

$$
\frac{h c}{\lambda}-\frac{h c}{\lambda^{\prime}}=\frac{h^{2}}{\lambda^{2} m_{e}}
$$

where $\lambda$ is the initial wavelength of the incoming photon, $\lambda^{\prime}$ the wavelength of the reemitted photon and $h$ the Planck constant.

Solving this equation gives:

$$
\delta \lambda\left(\lambda m_{e} c-h\right)=h \lambda
$$

Since $h \ll \lambda m_{e} c$ 


$$
\delta \lambda=\frac{h}{m_{e} c}=2.42 \times 10^{-12} \mathrm{~m}
$$

As the photons travel through the IGM they are constantly absorbed and re-emitted and on each interaction their wavelength increases by $h / m_{e} c$. Galaxies twice as far away, travel twice as far through the IGM, make twice as many interactions and thus suffer twice the redshift.

However, it is not as simple as that since the number of collisions depends upon the collision cross-section and this in turn depends upon the wavelength of the photon. The collision cross-section, $\sigma$ for a photon-electron interaction where the photon is absorbed can be found from low energy X-rays interacting with matter [38]-[40].

$$
\sigma=2 r_{e} \lambda f_{2}
$$

where $f_{2}$ is one of two "scattering factors" dependent upon the number of electrons in the atom and tends to equal that number for atoms of low atomic number (as the number of electrons in an atom increase they start to shield each other and so $f_{2}$ is less). For Hydrogen $f_{2}$ has values between 0 and 1 whilst for Helium $f_{2}$ has values between 0 and 2. For resonant absorption where the frequency of the incoming photon is equivalent to an energy level difference, $f_{2}=1$ and the photon is not re-emitted. If the frequency of the incoming photon is well away from any resonant frequency in the atom $2 r_{e} \lambda_{2}=0$ meaning the photon is not absorbed but re-emitted. Collision cross-sections are basically probabilities.

Consequently we see that the collision cross-section for photo-absorption consists of the Probability of the electron absorbing the photon $\left(2 r_{e} \lambda_{2}\right)$ multiplied by the probability of the electron not re-emitting it $\left(f_{2}\right)$. In New Tired Light we are interested in the collision cross-section (or probability) for transmission which is:

$$
\sigma=2 r_{e} \lambda\left(1-f_{2}\right)
$$

and since $f_{2}$ is zero when we are well away from resonance the collision cross-section for New Tired Light is:

$$
\sigma=2 r_{e} \lambda
$$

The mean free path of the photons is $\left(n_{e} \sigma\right)^{-1}$ or $\left(2 n_{e} r_{e} \lambda\right)^{-1}$ where $n_{e}$ is the number density of electrons in the IGM and since the photon is redshifted at each interaction the mean free path will get shorter and shorter as it travels through space.

If the initial wavelength is $\lambda$, it will be $\left(\lambda+h / m_{e} c\right)$ after one interaction, $\left(\lambda+2 h / m_{e} c\right)$ after two interactions, $\left(\lambda+3 h / m_{e} c\right)$ after three and so on.

The sum of the mean free paths is equal to $d$, the total distance travelled.

$$
\begin{aligned}
& d=\left[2 n_{e} r_{e} \lambda\right]^{-1}+\left[2 n_{e} r_{e}\left(\lambda+h / m_{e} c\right)\right]^{-1}+\left[2 n_{e} r_{e}\left(\lambda+h / m_{e} c\right)\right]^{-1} \\
&+\cdots+\left[2 n_{e} r_{e}\left(\lambda+(N-1) h / m_{e} c\right)\right]^{-1} \\
& \sum_{x=0}^{N-1}\left\{\lambda+x\left(\frac{h}{m_{e} c}\right)\right\}^{-1}=2 n_{e} r_{e} d
\end{aligned}
$$

And since $N$ is large and $h / m_{e} c$ is small this approximates to: 


$$
\begin{aligned}
& d= {\left[2 n_{e} r_{e} \lambda\right]^{-1}+\left[2 n_{e} r_{e}\left(\lambda+h / m_{e} c\right)\right]^{-1}+\left[2 n_{e} r_{e}\left(\lambda+h / m_{e} c\right)\right]^{-1} } \\
&+\cdots+\left[2 n_{e} r_{e}\left(\lambda+(N-1) h / m_{e} c\right)\right]^{-1} \\
& \sum_{x=0}^{N-1}\left\{\lambda+x\left(\frac{h}{m_{e} c}\right)\right\}^{-1}=2 n_{e} r_{e} d \\
& \int_{0}^{N-1}\left\{\lambda+x\left(\frac{h}{m_{e} c}\right)\right\}^{-1} \mathrm{~d} x=2 n_{e} r_{e} d
\end{aligned}
$$

Giving:

$$
N=\lambda\left(\left(m_{e} c / h\right) \exp \left(2 n_{e} h r_{e} d / m_{e} c\right)+1-\lambda\left(m_{e} c / h\right)\right)
$$

The total increase in wavelength, $\Delta \lambda=N \delta \lambda$ ie $\mathrm{Nh} / \mathrm{m}_{e} \mathrm{C}$

$$
\Delta \lambda=\lambda \exp \left(2 n_{e} h r_{e} d / m_{e} c\right)+h / m_{e} c-\lambda
$$

The redshift, $z$ is $z=\Delta \lambda / \lambda$

$$
z=\exp \left(2 n_{e} h r_{e} d / m_{e} c\right)+h /\left(m_{e} c / \lambda\right)-1
$$

As $h /\left(m_{e} c / \lambda\right)$ is small compared to the other values $\left(=2.42 \times 10^{-12} \lambda^{-1}\right)$ for all wavelengths below $\mathrm{X}$ ray we can neglect it. N.B. for $\mathrm{X}$ ray and above this classical approach fails as the recoil approaches the speed of light and so relativistic effects need to be considered.

$$
z=\exp \left(2 n_{e} h r_{e} d / m_{e} c\right)-1
$$

Since $v=c z$ and in the Hubble Law $v=H d$

$$
H=(c / d)\left\{\exp \left(2 n_{e} h r_{e} d / m_{e} c\right)-1\right\}
$$

For small astronomical distances we use the approximation $\mathrm{e}^{x} \approx 1+X$

$$
H=2 n_{e} h r_{e} / m_{e}
$$

Or:

$$
z=\exp (H d / c)-1
$$

which is the Tired Light relationship proposed by Zwicky in 1929 [41].

Note that the exponential function is linear for small values i.e. for local galaxies, $Z=$ $H d / c$ or $v=H d$.

With the value of the average electron number density given from the dispersion value earlier $n_{e}=0.5 \mathrm{~m}^{-3}$ we can predict the value of the Hubble constant by New Tired Light.

$$
h=6.63 \times 10^{-34} \mathrm{~J} \cdot \mathrm{s} ; r_{e}=2.82 \times 10^{-15} \mathrm{~m} ; m_{e}=9.1 \times 10^{-31} \mathrm{~kg}
$$

giving $2.05 \times 10^{-18} \mathrm{~s}^{-1}$ or $63 \mathrm{~km} / \mathrm{s}$ per Mpc which compares favourably with presently accepted values [26].

\section{A.2. The Cosmic Microwave Background Radiation}

The recoiling electron will interact with the other charges in the plasma and the kinetic 
energy gained by recoil will be emitted in the form of secondary radiation [42]. Since the interactions are non-relativistic it is a simple matter to find the wavelength of these secondary photons.

Momentum of photon, $p=h / \lambda$ and Kinetic energy $E_{k}=p^{2} / 2 m$ giving:

$$
\lambda_{\text {CMB }}=2 m_{e} \lambda^{2} c / h
$$

Light of wavelength $5 \times 10^{-7} \mathrm{~m}$ produces secondary photons from the recoil of 0.21 $\mathrm{m}$. The peak of the $C M B$ is at a wavelength of $2.1 \times 10^{-3} \mathrm{~m}$ [24] [25]. Secondary photons at this wavelength are produced by the redshifting of a photon of wavelength $5 \times$ $10^{-8} \mathrm{~m}$ i.e. a UV photon. NB Plasma radiates Black body radiation hence the radiation formed by the redshifting of photons is not only in the microwave but is also black body in nature [43].

This leads to a possible test of NTL as this $C M B$ will also be redshifted. If visible light/UV/IR are redshift and produce a peak in the microwave region then the $C M B \mathrm{R}$ will also be redshifted. Photons at the peak of the $C M B R$ have a wavelength of $2.1 \times$ $10^{-3} \mathrm{~m}$ and we can use our formula to calculate the secondary radiation given off when these photons are radiated. The peak will be at $\lambda=3.64 \times 10^{6} \mathrm{~m}$ and frequency $82 \mathrm{~Hz}$. This is the ELF range and used by submarines to communicate. Consequently if we look to outer space in this region we should find an omnidirectional non seasonal signal peaking at $82 \mathrm{~Hz}$.

\section{Appendix B. Why It Cannot Be Compton Scatter That Causes the Redshifts}

Some researches confuse NTL with the Compton Effect and this is definitely not the case. In Compton scatter the photons are assumed to be absorbed and re-emitted instantaneously as there is no mechanism within Compton Scatter that the "free" electron can store the energy during any delay. Hence, to conserve momentum, the photon goes off to one side whilst the electron goes off to the other. There is no possibility of a loss in energy in the forward direction and hence a redshift along the line of sight as it cannot recoil in this direction since absorption and re-emission take place instantaneously. The light is scattered as it travels along and the image would blur. This is not seen in observations.

In NTL the electrons are not free but interact loosely with the other charges in the plasma. If an electron is displaced in the forwards direction, the region in front of it becomes overall slightly negative since it has gained and an electron and the region behind it has become slightly positive as an electron has moved away. Restoring forces act on the electron and thus it performs SHM. Any electron that can perform SHM can absorb and re-emit photons. However, since there is a delay, between absorption and re-emission the energy of the photon is transferred to vibrational energy of the electron with a tiny amount going to Kinetic energy of the recoiling electron. Whilst the vibrational energy is re-emitted as a new photon the KE has been lost to the photon and is emitted as a secondary photon that forms the $C M B$. 
Submit or recommend next manuscript to SCIRP and we will provide best service for you:

Accepting pre-submission inquiries through Email, Facebook, LinkedIn, Twitter, etc. A wide selection of journals (inclusive of 9 subjects, more than 200 journals)

Providing 24-hour high-quality service

User-friendly online submission system

Fair and swift peer-review system

Efficient typesetting and proofreading procedure

Display of the result of downloads and visits, as well as the number of cited articles

Maximum dissemination of your research work

Submit your manuscript at: http://papersubmission.scirp.org/ 